\title{
Review \\ Optical soliton in dielectric fibers and self-organization of turbulence in plasmas in magnetic fields
}

\author{
By Akira HASEGAWA $* 1, * 2, \dagger$
}

(Communicated by Sogo OKamurA, M.J.A.)

\begin{abstract}
One important discovery in the twentieth century physics is the natural formation of a coherent or a well-ordered structure in continuous media, in contrary to degradation of the state as predicted earlier from the second law of thermodynamics. Here nonlinearity plays the essential role in its process. The discovery of soliton, a localized stable wave in a nonlinear and dispersive medium and the self-organization of fluid turbulence are of the major examples. A soliton is formed primarily in one-dimensional medium where the dispersion and nonlinearity play the essential role. Here the temporal evolution can be described by an infinite dimensional Hamiltonian system that is integrable. While a self-organization appears in an infinite dimensional non-Hamiltonian (or dissipative) system where more than two conservative quantities exist in the limit of no dissipation. In this manuscript, by showing examples of the optical soliton in dielectric fibers and self-organization of turbulence in a toroidal plasma in a magnetic field, we demonstrate these interesting discoveries. The manuscript is intended to describe these discoveries more on philosophical basis with some sacrifice on mathematical details so that the idea is conveyed to those in the wide area of sciences.
\end{abstract}

Keywords: soliton, optical soliton, plasma turbulence, self-organization, Hasegawa-Mima equation, zonal flow

\section{Introduction}

In a system having an infinite degrees of freedom, the second law, the law of entropy increase, is the major discovery in thermodynamics. The law is so fundamental that the direction of time is defined as that of the increase of entropy. Although the second law was originally defined as a degradation of energy, it is now interpreted more generally as degradation of a coherent state (or increase of disorder), as a result of development of the information theory. Even in a system with infinite degrees of freedom (often referred to as an infinite dimensional (Hamiltonian) system), if the temporal evolution is described by a linear partial differential equation, it is exactly integrable by

*1 Professor Emeritus, Osaka University, Osaka, Japan.

$* 2 \quad$ Recipient of Japan Academy Prize in 2008.

$\dagger$ Correspondence should be addressed: A. Hasegawa, 403 Awataguchi Sanjobocho, Higashiyama-ku, Kyoto 605-0035, Japan (e-mail: akirah@solitoncomm.com). means of the Fourier transformation, the time evolution is precisely predictable and thus results in no change of entropy. As a result, it has been expected that nonlinearity of the system is responsible to the increase of entropy. We show, however, the nonlinearity is also responsible for formation of ordered structures.

In the latter half of the twentieth century, thanks to the development of high-speed computers, time evolution of nonlinearly interacting many body systems and infinite dimensional nonlinear evolution equations (nonlinear partial differential equations) have been extensively studied. Although, the nonlinearity by and large was found to be responsible to the entropy production, it was discovered that a large class of nonlinear evolution equations with a Hamiltonian structure is precisely integrable and the time asymptotic behavior can be described by a set of stable localized nonlinear waves (called solitons) and linear dispersive waves. In addition, it was discovered that even in non- 
Hamiltonian systems, a random initial excitation can lead to formation of a statistically ordered state as exemplified in two dimensional Navier-Stokes turbulence.

In this manuscript, as potential examples of these phenomena, we present generation of optical solitons in dielectric fibers and formation of zonal flow in magnetized cylindrical plasma. These examples are interesting not only as examples of generation of ordered structure in nonlinear systems, but also in their applications to real world problems. Optical solitons are playing the crucial role in ultra-high-speed information transfer over cross continental distances, while the generation of zonal flows is expected to control plasma loss by inhibiting turbulent transport of plasma particles to the container wall.

\section{Discovery of optical soliton}

During the early stage of development of optical communications over dielectric fibers (later called optical fibers), fiber dispersion, whereby optical information carried by different frequency components propagates at different speeds, has been a major concern since it leads to distortion of an optical pulse, thus the optical information carried by it. In 1973, when the fiber based optical communication is still in its premature stage, the author derived the master evolution equation for information transfer in optical fibers ${ }^{1)}$ by taking into account of the (group velocity) dispersion and the Kerr nonlinearity of the fiber and demonstrated that it has a solitary wave solution for a range of parameters of practical interests. F. D. Tappert ${ }^{1)}$ checked the stability of the solution numerically and confirmed its stability and showed the solution has the soliton property. If the fiber loss is compensated for by proper optical amplifiers, the evolution equation for the properly normalized complex light envelope (or optical information), $q(Z, T)$, in a coordinate moving at the linear group velocity, satisfies the nonlinear Schrödinger equation of the form, ${ }^{1)}$

$$
\frac{\partial q}{\partial Z}=\frac{i}{2} \frac{\partial^{2} q}{\partial T^{2}}+i|q|^{2} q
$$

Here, the first term on the right hand side represents the dispersion effect, while the second term, the nonlinear effect. In this equation, $T$ is the time normalized to the pulse width, $Z$ is distance normalized to the dispersion distance for the corresponding pulse (distance over which the pulse width increases by a factor of 2 due to the linear group velocity dispersion of the fiber). The complex amplitude $q$ is normalized to the intensity that gives a nonlinear phase shift of $\pi$ over the dispersion distance. In other words, $q$ takes a value on the order of unity if the distance for nonlinear phase shift is comparable to the dispersion distance. The nonlinear phase shift occurs due to the nonlinear dielectric response that leads to a small change of index of refraction of the fiber (called the Kerr effect). The nonlinear change of the index of refraction (which increases in proportion to the light intensity) is very small, on the order of $10^{-12}$ for a light intensity of $1 \mathrm{~mW}$ in the fiber. However, since the real distance of communication, which is on the order of $10^{6} \mathrm{~m}$, if this distance is measured in terms of the light wavelength, $10^{-6} \mathrm{~m}$, it becomes very large, on the order of $10^{12}$. Thus, this small change of the index of refraction produces an appreciable effect on the pulse distortion over this distance. A modern fiber is designed to reduce the dispersion effect and the dispersion distance is on the order of the standard transmission distance of $10^{6} \mathrm{~m}$. Thus the complex amplitude in Eq. [1] is in fact has a value on the order of unity, and the nonlinearity and the dispersion play equally important role. However, this fact has not been recognized widely until twenty years later, when a fiber based long distance optical communication became technically feasible owing to the development of optical amplifiers and low loss dispersion free fibers.

\section{Concept of a soliton, a brief review}

What is interesting about Eq. [1] is the fact that this equation is integrable ${ }^{2)}$ and falls into a class of nonlinear evolution equations where the asymptotic solution for any localized initial wave structures can be described by a set of stable localized waves called solitons. The term "soliton" was first coined by Zabusky and Kruskal ${ }^{3)}$ in 1965 when, in the course of numerical solution of the Korteweg-deVries equation, ${ }^{4)}$ they discovered numerically that pulse-like solitary waves were generated from a sinusoidal initial condition and they interacted elastically each other. The KortewegdeVries equation is a model evolution equation that was derived much earlier to describe the water 
surface wave with dispersion and depth dependent nonlinearity. The soliton was recognized its significance later when it was discovered to play a role of fundamental solution to certain class of nonlinear dispersive evolution equations. The pioneering work that has demonstrated this was done by Gardner, Greene, Kruskal and Miura ${ }^{5}$ in their mathematical solution of the Korteweg-deVries equation by making use of direct and inverse scattering method used in quantum mechanics. The method can be described as follows; Take an eigenvalue equation with the eigenvalue given by $\lambda$. Take an operator $L$ associated with the eigenvalue that contains the wave amplitude, $q$, such as one given by Eq. [1],

$$
L \psi=\lambda \psi
$$

Assume that the evolution of the eigen function, $\psi$, is determined by another operator $M$ that contains also the wave amplitude, $q$,

$$
\frac{\partial \psi}{\partial Z}=M \psi
$$

We now require that the eigenvalue $\lambda$ is invariant in the evolution in the coordinate $Z$. The compatibility condition imposed on the operators $L$ and $M$ that enables invariance of the eigenvalue gives,

$$
\frac{\partial L}{\partial Z}=M L-L M
$$

With a proper choice of $L$ and $M$, Eq. [4] may turn out to be identical to the evolution equation that we are interested in solving, such as Eq. [1], then the evolution equation is solvable by means of direct and inverse scattering method for a prescribed initial value of $q=q(0, T)$ with the help of Eqs. [2] and [3]. The operator pair, $L$ and $M$ is called the Lax pair after the discoverer of this method. $\left.{ }^{6}\right)$ The asymptotic solution is then given by $\mathrm{n}$ set of solitons whose characteristics (such as amplitude and phase) are given by the invariant eigenvalues, $\lambda$ and dispersive waves. The number of eigenvalues is determined by the initial value of $q(0, T)$ of Eq. [2] $q(0, T)$ plays a role of potential function if Eq. [2] has a structure of the Schrödinger equation. Number of eigen values depends on the depth of the potential, and this number determines the number of solitons that emerges from $q(0, T)$ in the course of evolution of $q$ in $Z$. The evolution of $q(Z, T)$ is given by Eq. [4], an example of which is given by Eq. [1].
Zakharov and Shabat ${ }^{7)}$ discovered the pair of the Lax operators $L$ and $M$ such that Eq. [4] gives the nonlinear Schrödinger equation, [1]. This discovery warrants the fact that any pulse introduced to an optical fiber whose loss is compensated for by optical amplifiers evolves to a soliton (or a set of solitons) as it propagates over several dispersion distances whether one likes or not.

The fact that a certain class of nonlinear evolution equations can be solved by the inverse scattering method and that the solution can be described by a set of $n$ solitons is a remarkable discovery in the twentieth century applied mathematics and physics. Here solitons play a role of Fourier modes for a linear evolution equation, thus solitons are often quoted as nonlinear Fourier modes. Through this discovery, it is recognized that a soliton is not merely a stable nonlinear wave but is the elementary wave in nonlinear evolution equation whose role is equivalent to the Fourier mode in a linear evolution equation.

\section{Entropy law and Hamiltonian systems}

The integrability (meaning to be possible to establish the exact causal relation between the initial state and a state later in time on in distance) of an evolution equation for an infinite dimensional system as described here is closely related to that in Hamiltonian dynamics. In order to see the relationship of a soliton system (which has an exact causal relation with the initial condition) to the entropy law of thermodynamics that was also developed for infinite dimensional systems, let us discuss here how the present result is compatible with the second law of thermodynamics. The second law was developed for an infinite dimensional system that is not integrable where the only constant of motion (in the absence of dissipation) is the total energy. In this law, the only plausible result is that the evolution obeys the law of increase of entropy, or the degradation of energy. The causal relation between the initial condition and the final state of the system is lost and the system is believed to approach to the state of the maximum entropy.

The hint to the relation between integrable system and non-integrable system was discovered also through numerical studies made for finite dimensional evolution equations. To demonstrate this point, let us review the classic understanding 
of evolution of many body systems. It has been known that a two body system is integrable but a three (or more) body systems are not. What does this mean?

A classical two-body system is the motion of two point particles in a gravitational field (or in an electrostatic field for charged particles). For simplicity we assume that one particle has a mass infinitely larger the other particle and the relevant motion is that of a particle with lighter mass. The motion has only two degrees of freedom in the plane determined by the initial velocity of the lighter particle; we recognize that the motion has two constants of motion, one the total energy, the Hamiltonian itself, and the other, the angular momentum. The latter conservation comes from the point symmetry of the gravitational force. Thus the number of the degrees of freedom and that of the constants of motion match and as a result, the motion of the particle is exactly integrable. If the masses of the two particles are comparable, the system is still integrable by describing the motions of the particles in the frame of the center of gravity. However, a three-body system is known not to be integrable because the degrees of freedom exceed the number of constants of motion. We note also that a single charged particle in a dipole magnetic field is also not integrable since the degree of freedom of the motion is intrinsically three-dimensional there and there are only two constants of motion, energy and the canonical angular momentum. The latter comes from the axis symmetry of the dipole field. Since a dipole magnetic field is the simplest form of a magnetic field, it is interesting to note that the motion of a charged particle in any magnetic field is intrinsically not integrable.

During the later part of the twentieth century, extensive numerical studies have been performed to solve many body problems that have been known not to be integrable such as the three-body problem. Even if a system is not integrable based on the fact that the degrees of freedom are more than the number of constants of motion, it is expected that such a system may be solvable numerically. It is in fact numerically solvable but it was found that the motion of these particles was unstable in that the temporal evolution of the trajectories starting from infinitely small different initial conditions could diverge exponentially in time in a limited area in its phase space. The meaning of non-integrable
Hamiltonian system was thus identified for the first time numerically in the twentieth century. It should be noticed that a chaotic solution appears only in a limited area in phase space of the motion, thus the system is only partially unstable in the limited area of the phase space and integrable in other area. A nonlinear system in which the solution can be unstable in this sense is defined as chaotic. If a Hamiltonian system is chaotic, the entropy can increase in a limited area in phase space, even for a system of a finite number of degrees of freedom because the uncertainty of the solution increases if the result is averaged over different initial conditions. Thus the entropy law is justified even in a Hamiltonian system (non-dissipative system) with a small number of degrees of freedom. From this thought experiment, one can identify the meaning of the entropy law of thermodynamics for a Hamiltonian system.

Now, how the infinite dimensional system of evolution equation (that is integrable to form solitons) is compatible with the entropy law? The answer is that a nonlinear evolution equation that is integrable has infinite number of conservations, thus the degrees of freedom matches with the number of constants of motion. The soliton system is similar to the two body system in that both systems are Hamiltonian systems in which the number of constants of motion is the same as that of the degrees of freedom. The discussion presented here is helpful to the understanding of the selforganization of turbulence that will be described in the subsequent sections.

\section{Gibbs distribution in a system having additional constraint}

Turbulence in continuous media has been known to self-organize if the system has an independent constant of motion(s) in addition to the energy (in the limit of no dissipation). As was described in the previous section, integrability of a nonlinear evolution equation is closely related to the presence of constants of motion in addition to the Hamiltonian itself. As a result it is expected that if there exist additional constants of motion even in an infinite dimensional system such as a continuous medium, the temporal evolution would not result in a simple increase of entropy. A wellknown example of this is the two dimensional Navier-Stokes turbulence. Here, in addition to the 
energy, which is represented by the integrated square of the velocity field, $W=1 / 2 \int \mathbf{v}^{2} d V$, the integrated square of the vorticity called enstrophy, $U=1 / 2 \int(\nabla \times \mathbf{v})^{2} d V$, is also conserved in the absence of dissipation. The term "enstrophy" should not be confused with the term "entropy". If one constructs the Gibbs distribution by requiring maximizing entropy, with constraints of constant energy and of enstrophy, it will be given by

$$
P=\text { const } \times \exp (-\alpha W-\beta U) .
$$

Here alpha and beta are the Lagrange multipliers representing the constraints for energy and enstrophy. In the wavenumber Fourier space, the spectral density of enstrophy $U_{k}$ is related to that of energy density through, $U_{k}=k^{2} W_{k}$, the Gibbs distribution gives the expectation for the energy density in the wavenumber space,

$$
\left\langle W_{k}\right\rangle=\frac{1}{\alpha+\beta k^{2}} .
$$

In the absence of the constraint of enstrophy, Eq. [6] gives,

$$
\left\langle W_{k}\right\rangle=\frac{1}{\alpha}=\kappa T
$$

which gives the well-known equipartition law of energy with alpha $=1 / \kappa T, \kappa$ being the Boltzmann constant. However, in the presence of the constraint of enstrophy conservation, the energy density $W$ can take a negative value if the Lagrange multiplier is negative. This indicates that such a system can have an effectively negative temperature and the energy spectrum might concentrate at a specific value of wavenumber. This argument is an indicative of a presence of a thermodynamical equilibrium that does not obey the classic equipartition law and of a nontrivial equilibrium state where the entropy is still maximized. If the spectral distribution of the energy has a peak in certain value of the wavenumber at the maximum entropy, it is expected that the thermodynamical equilibrium may have certain ordered structure around at the maximum value of energy spectral density.

The Navier-Stokes equation is a macroscopic equation that describes evolution of the velocity field of fluids and charge neural gases. Thus it always accompanies dissipation (due to fluid viscosity) and Gibbs distribution becomes irrelevant.
So let us discuss how the self-organization may take place in two dimensional fluids.

\section{Two dimensional Navier-Stokes turbulence and inverse cascade of energy spectrum}

The Navier-Stokes equation is derived from the fluid equation of motion with constant mass density, $\rho$, and the kinematic viscosity, $\nu$,

$$
\frac{d \mathbf{v}}{d t}\left[=\frac{\partial \mathbf{v}}{\partial t}+(\mathbf{v} \cdot \nabla) \mathbf{v}\right]=-\nabla T+\nu \nabla^{2} \mathbf{v}
$$

for an incompressible fluids, the velocity field, $\mathbf{v}$, satisfies,

$$
\nabla \cdot \mathbf{v}=0
$$

The incompressibility applies to slowly moving fluids and gases and Eq. [9] plays the role of the equation of state. This equation of state does not necessarily warrants that the mass density is constant. However, if the mass density is uniform, it can be treated as constant and the fluid equation of motion can be described as the evolution of the velocity field, [8]. If we further assume that the fluid motion is constrained in a two dimensional plane, $x$ and $y$, the equation of motion can be reduced to that of the vorticity field vector, $\Omega=(\nabla \times \mathbf{v})$, directed in the $\mathrm{z}$ direction. i.e., by taking the curl of [8], we have,

$$
\frac{d \Omega}{d t}\left[=\frac{\partial \Omega}{\partial t}+(\mathbf{v} \cdot \nabla) \Omega\right]=\nu \nabla^{2} \Omega .
$$

Furthermore, in two dimensions the velocity field and vorticity may be expressed by a scalar stream function, $\psi$.

$$
\begin{aligned}
\mathbf{v} & =-\nabla \times \psi \widehat{\mathbf{z}}=-\nabla \psi \times \widehat{\mathbf{z}} \\
\Omega & =\nabla^{2} \psi \widehat{\mathbf{z}},
\end{aligned}
$$

where $\widehat{\mathbf{z}}$ is the unit vector in the $\mathrm{z}$ direction. Note that $\mathbf{v}$ is tangential to $\nabla \psi$, the fluid moves on the $\psi=$ constant contour. The evolution equation for the vorticity [11] can then be written in terms of the stream function only,

$$
\frac{\partial}{\partial t} \nabla^{2} \psi-(\nabla \psi \times \hat{\mathbf{z}}) \cdot \nabla\left(\nabla^{2} \psi\right)-\nu \nabla^{4} \psi=0,
$$

where $\nabla$ is the two dimensional gradient operator,

$$
\nabla=\frac{\partial}{\partial x} \widehat{\mathbf{x}}+\frac{\partial}{\partial y} \widehat{\mathbf{y}}
$$

One first notes that linearization of equation [12] 
gives a purely damped mode whose dispersion relation is given by $\omega=-i \nu k^{2}$, so no quasi-particles (or eigen modes) are involved in this system. This fact indicates that the so called weak turbulence theory does not apply here. Second, if the viscosity is small, a mode excited in this system is highly nonlinear, that is, the time evolution is determined by the second term in [12]. The ratio of the second term (nonlinear term) to the third term (linear viscous term), $V /(\nu L)$ (where $L$ is the characteristic scale length and $V$ is the magnitude of the flow velocity) is called the Reynolds number and gives a measure of the strength of "nonlinearity" of the system. If the Reynolds number is large, spatial Fourier modes rapidly cascade two-dimensionally to other Fourier modes and a turbulent state results. The evolution of the turbulent spectra as a result of the nonlinear term is called spectrum cascade. Thirdly, the two dimensional NavierStokes equation has two independent conservations in the absence of dissipation, the total energy, W, and the total enstrophy, U, as stated earlier. The conservation of the total energy can be shown by constructing a scalar product of $\mathbf{v}$ with Eq. [8] and integrating over the two dimensional volume, $V$

$$
\begin{aligned}
\frac{\partial}{\partial t} W & \equiv \frac{\partial}{\partial t} \int\left(\frac{v^{2}}{2}\right) d V \\
& =-\int\left(\frac{v^{2}}{2}+T\right) \mathbf{v} \cdot d \mathrm{~S}=0 .
\end{aligned}
$$

Here the velocity field normal to the boundary surface $\mathbf{S}$ may be assumed to vanish. Similarly the conservation of the enstrophy can be constructed from the scalar product of $\boldsymbol{\Omega}$ and Eq. [10], as

$$
\frac{\partial}{\partial t} U \equiv \frac{\partial}{\partial t} \int \frac{\Omega^{2}}{2} d V=-\int \frac{\Omega^{2}}{2} \mathbf{v} \cdot \mathbf{d} \mathbf{S}=0 .
$$

We note here that in three dimensions there is an additional term on the right-hand side, $\int \Omega$. $(\Omega \cdot \nabla) \mathbf{v} d V$, which invalidates the enstrophy conservation.

\section{Turbulence spectra and inverse cascade}

Weakly dissipative fluid having a large Reynolds number is fully nonlinear and its mathematical description is very difficult, if not impossible. One plausible argument in deriving the turbulent spectra in such a fluid was made by Kolmogorov. ${ }^{8)}$ He argued that when a fluid having a large Reynolds number is excited, the turbulent energy spectrum will cascade in the wavenumber space owing to the convective nonlinear term (the second term on the right hand side of [8]) smoothly to large wavenumber regions where it will be dissipated by the viscous damping. He defined the energy spectrum in the cascading region as the inertial range spectrum. Let us show here how the inertial range spectrum is obtained in three dimensional Navier Stokes fluids.

If we write the Fourier amplitude of the velocity field as $\mathbf{v}_{\mathbf{k}}$, the rate at which the spectrum cascades by the convective nonlinear term is given by $\mathrm{kv}_{\mathrm{k}}$. We like to derive the omni-directional energy spectral density, $W(k)$, which is defines as such $\int W(k) d k$ gives the total energy, where $k=$ $(\mathbf{k} \cdot \mathbf{k})^{1 / 2}$; hence $W(k) k$ has the dimension of $v_{k}^{2}$. The inertial range is obtained by requiring that the cascading rate of the energy spectrum $\rho k v_{k} v_{k}^{2}$ is constant and given by the dissipation rate $\varepsilon$ at large wave numbers where $\rho$ is the mass density. Writing $v_{k}=[k W(k)]^{1 / 2}$, the energy spectral density in the inertial range is given by

$$
W(k)=C\left(\frac{\varepsilon}{\rho}\right)^{2 / 3} k^{-5 / 3}
$$

where $\mathrm{C}$ is a universal, dimensionless constant. Equation [16] represents the famous Kolmogorov spectrum that has been experimentally observed in various fluids.

Now let us consider what will be the inertial range spectra for two dimensional Navier-Stokes turbulence. As was shown, two dimensional fluids have the enstrophy as an additional conserved quantity. As a result, one expects two inertial range spectra, one for the energy and the other for the enstrophy. Since the enstrophy density is given by $k^{2} v_{k}^{2}$, the inertial range of enstrophy requires that

$$
\rho k v_{k} k^{2} v_{k}^{2}=\varepsilon^{\prime}(=\text { const }) .
$$

Thus, by expressing $v_{k}$ in terms of energy density, the inertial range spectrum is given by

$$
W(k)=C^{\prime}\left(\frac{\varepsilon^{\prime}}{\rho}\right)^{2 / 3} k^{-3} .
$$

This result shows that the spectral density of the energy for the inertial range of the enstrophy cascade differs from that of the energy cascade and given by $k^{-3}$ in contrast to that of the energy cascade, $\mathrm{k}^{-5 / 3}$. 

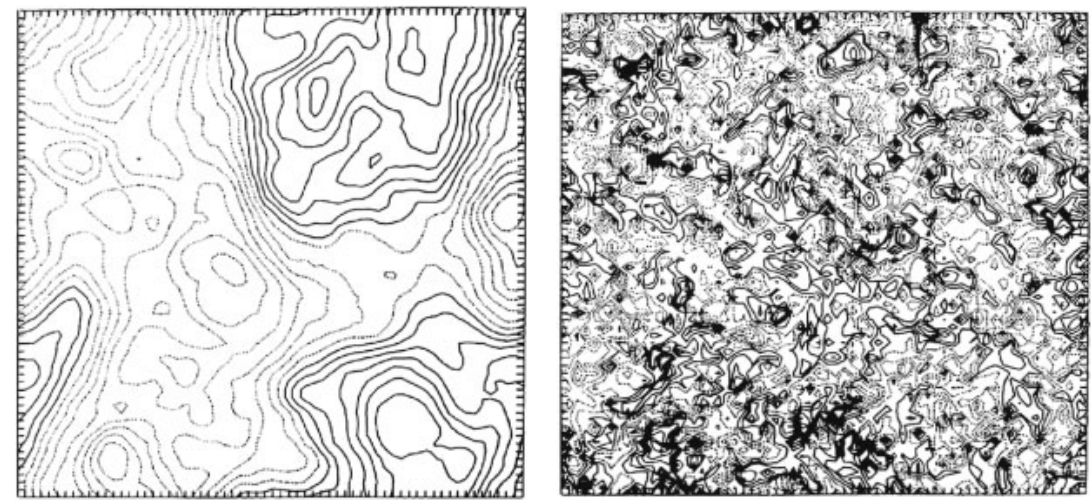

Fig. 1. Energy contours (stream function) (left) and vorticity contours (right) of fully developed two dimensional Navier-Stokes turbulence obtained by Lilly. ${ }^{11)}$ Owing to the condensation of the energy spectrum to the smallest wave-numbers, large scale stream function is generated, while the cascading of enstrophy generates small structure eddies.

Now, how can two types of energy spectra are possible in two-dimensional Navier-Stokes turbulence? Consider that the turbulence is excited by an injection of energy at a wavenumber $k=k_{s}$. The enstrophy will also by injected at the same wavenumber. Since the enstrophy spectrum is proportional to $k^{2}$ times energy spectrum, it will be more effectively dissipated by viscosity at large wave numbers. This implies that the enstrophy cascades to larger wave numbers at $k>k_{s}$, where the energy spectral density will obey $W(k) \sim k^{-3}$ rule of Eq. [18]. In fact Kraichnan ${ }^{9)}$ showed that in this regime of wave numbers, there is no energy cascade. As a result energy spectrum can cascade only to smaller wavenumber spaces and establishe the Kolmogorov spectrum of $k^{-5 / 3}$. In this regime, he showed that there would be no enstrophy cascade and the energy spectrum cascades to smaller wave numbers. He called this process as the inverse cascade in contrast to the three dimensional turbulence where the energy cascades to larger wave numbers. The author ${ }^{10)}$ showed that the decay of a mode to two wave numbers based on the nonlinear terms of the Navier-Stokes equation with the constraints of the conservation of both energy and enstrophy, results in spectrum condensation at $k=0$, without the use of the inertial range and selective dissipation argument of Kraichnan.

The preceding arguments produce a very interesting and unexpected result in the two-dimensional Navier-Stokes turbulence. Since the energy cascades to smaller wave-numbers, the turbulence will create a larger and larger structures that the system allows and eventually some ordered structures may arise. Such a process is a form of selforganization. Computer simulations of two dimensional Navier-Stokes turbulence elucidate the selforganization nature most clearly. Figure 1 shows the result of a computer simulation made by Lilly ${ }^{11)}$ in 1969. Two-dimensional Navier-Stokes turbulence is excited by a source at a medium wavenumber. As a result of inverse cascade of the energy spectrum, the stream function (which corresponds to energy contours) exhibits formation of large structures as shown in the left figure. In contrast, the vorticity contours exhibits formation of small structures as seen in the right figure as a result of normal cascade of enstrophy.

Here, it may be in order to discuss the entropy argument. How this type of self-organization is compatible with the entropy law of thermodynamics? Where does the disorder increases while seemingly smooth and ordered structure arises in stream function? Answers to these questions can be seen in the right side figure of Fig. 1, where the vorticity contours reveals disordered structures. The second law of thermodynamics works in the increase of disorder in enstrophy! What is interesting here is that for human eyes, only steam function and not the vorticity is visible in fluids and as a result it appears as though the entropy decreased. The nature is just deceiving the human eyes. We may be happy to see ordered structures visible in human eyes even though disorder increases simultaneously in physical quantities invisible to our eyes. The analogy goes like this; if the secretary cleans up 
your desktop and the result looks neat, the messy information of the desktop has been transferred to the secretary's brain where the entropy has increased. But the brain of your secretary is invisible to you, while the desk top entropy has seemingly decreased. This is the process of self-organization. This result reminds us of a famous quotation by Chung-Tze of the 4th century BC, which says "People considers ladies Maochiang and Lichi as classic beauties, Seeing them, however, fish dive deeper, birds fly higher, and deer run away. Which of the four knows the true color of nature?"

\section{Electrostatic turbulence of plasmas in magnetic fields}

In reality fluid motion can not be constrained to two dimensional plane, thus the argument presented in the previous section, although it is indicative of very interesting phenomena, is a thought experiment that does not exist in a practical situation. In this section, we introduce behavior of plasmas in a magnetic field and demonstrate that its turbulence can be described by a model equation (called the Hasegawa-Mima equation ${ }^{12)}$ ) that has a property quite similar to the two dimensional Navier-Stokes turbulence. Although plasma motion in a magnetic field is three dimensional, confinement of the plasma by the magnetic field restricts the ion fluid motion primarily in the plane perpendicular to the magnetic field. While the electrons having a mass much lighter than ions can move freely in the direction of the magnetic field and their motion is intrinsically three-dimensional. However, the evolution of the electrostatic potential can be described in the coordinates perpendicular to the direction of the magnetic field.

A plasma is the forth state of matters in which atoms are ionized by high temperature and electrons and ions can move freely. It constitutes a conductive fluid and its behavior is quite complex in that microscopically it is a bunch of interacting charged particles, while macroscopically it behaves like fluids. The controlled nuclear fusion takes place for fuels in plasma state since the temperature required for fusion reaction is much higher than the ionization energy. A strong magnetic field is used to confine the plasma to achieve enough fusion reaction. It was found that plasmas confined by a toroidal magnetic field in a toroidal container exhibits turbulence in the microscopic scale (the scale of ion gyro-radius). Anomalous diffusion of the plasma across the confining magnetic field has been the main concern in achieving the controlled fusion reaction sufficient to produce enough energy output. Consequently, it is of fundamental importance to understand such plasma turbulence and discover a way (if any) to control the anomalous diffusion. The Hasegawa-Mima equation that Mima (at Osaka University) and the author derived in 1978 that describes such turbulence is simplest yet most fundamental evolution equation for plasmas in a strong magnetic field. It contains the feature of both microscopic and macroscopic plasma behavior where the evolution of the ion vorticity is described by electrostatic potential field, $\phi$,

$$
\begin{aligned}
& \frac{\partial}{\partial t}\left(\nabla^{2} \phi-\phi\right) \\
& \quad-[(\nabla \phi \times \widehat{\mathbf{z}}) \cdot \nabla]\left[\nabla^{2} \phi-\ln \left(\frac{n}{B}\right)\right]=0 .
\end{aligned}
$$

Here, $n$ and $B$ are spatially dependent plasma density and magnetic flux density, and $\nabla$ is the gradient operator perpendicular to the magnetic field which is directed to the $\mathrm{z}$ axis. This equation differs from the two-dimensional Navier-Stokes equation, [12], in two aspects. One the second term on the right hand side that originates from the electron motion in the $\mathrm{z}$ direction, and the other the fourth term that originates from the inhomogeneous background density and the magnetic flux density.

A very interesting aspect of this equation is that it has a property quite similar to the two dimensional Navier-Stokes equation, in particular it contains two independent conservations, the energy,

$$
\frac{\partial W}{\partial t} \equiv \frac{\partial}{\partial t} \int\left[(\nabla \phi)^{2}+\phi^{2}\right] d V=0,
$$

and the (potential) enstrophy,

$$
\frac{\partial U}{\partial t}=\frac{\partial}{\partial t} \int\left[(\nabla \phi)^{2}+\left(\nabla^{2} \phi\right)^{2}\right] d V=0 .
$$

This fact is a strong indicative of inverse cascade of turbulence in a plasma turbulence in a strong magnetic field. As a matter of fact, computer simulations based on an evolution equation that includes viscous dissipation as well as source of free energy due to pressure gradients and magnetic field curvature did reveal this feature. The simulation 
(a)

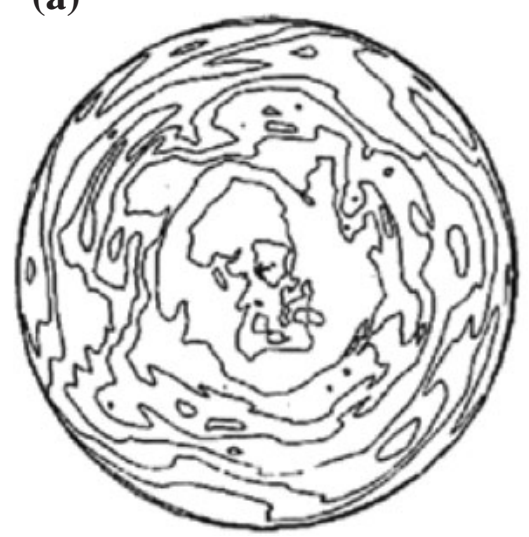

(b)

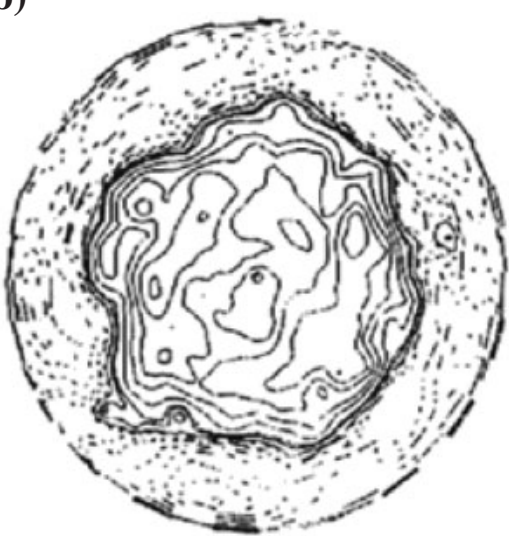

Fig. 2. Equipotential lines that also correspond to the stream function formed at the initial (a) and later (b) time in a cross-section of a toroidal plasma. Here solid (dotted) lines show positive (negative) equipotentials. It can be seen, after plasma turbulence is excited, a closed coaxial equipotential lines (that correspond to the stream function) are formed (right figure). This is a result of self-organization of turbulence. As a result plasma acquires zonal flow which tends to inhibit radial motion of convective eddies and helps to reduce anomalous diffusion. Simulation results obtained by Hasegawa and Wakatani. ${ }^{13)}$

was performed by Hasegawa and Wakatani ${ }^{13)}$ and a result is shown in Fig. 2.

What is shown here is the equipotential lines (solid (dotted) lines are for positive (negative) potentials) in the cross section of the cylindrical plasma. The magnetic field is directed perpendicular to the plane. The equipotential lines are equivalent to the stream function, that is, plasma flows along these lines. The inverse cascade process produces increased size of the turbulence structure and as a result, the equipotential lines are enlarged. The largest scale size of the equipotential line is limited by the cylindrical size of the plasma, but the constraint of the conservation of the total angular momentum inhibits the maximum size of the equipotential line to a size smaller than that of the cylindrical container. The spectral energy of the microscopic turbulence of the plasma is cascaded to macroscopic plasma flows and plasma rotates in the plane perpendicular to the magnetic field. The direction of the rotation is proportional to the radial electric field, $-\partial \phi / \partial r$. However the conservation of the angular momentum does not allow the plasma to rotate in one direction as a whole, so the plasma ends up rotating in one direction in the core region and to the opposite direction outside of the region. Therefore, a sheared azimuthally flow is established as a result of the inverse cascade. This remarkable result has been demonstrated in a number of experiments some twenty years after the derivation of the Hasegawa-Mima equation. Furthermore, the simulation result of Hasegawa and Wakatani ${ }^{13)}$ has indicated that convective cells that had been believed to cause the anomalous plasma diffusion are inhibited to move across cocylindrical equipotential line produced by the inverse cascade. This result is even more remarkable since it indicates that plasma turbulence can reduce anomalous diffusion contrary to the common belief. A similar phenomenon in fact can be seen in nature. Figure 3 is the picture of the Jovian atmosphere taken by the spacecraft, Voyager. The Jovian atmosphere is tinted by the sulfur gas emitted from the satellite Io. As a result, the zonal flows in the atmosphere are clearly visible. The generation of the zonal flow is believed as a consequence of the self-organization. ${ }^{14)}$ The famous red spot seen near the right bottom corner of the picture has been known to be stable for hundreds of years having been sandwiched by the zonal flow. The zonal flow is inhibiting the latitudinal motion of the red spot. As a matter of fact, the structure of the equation that describes atmospheric motion in a rotating planet is identical to the Hasegawa-Mima equation.

\section{Concluding remarks}

One important discovery in the 20th century 


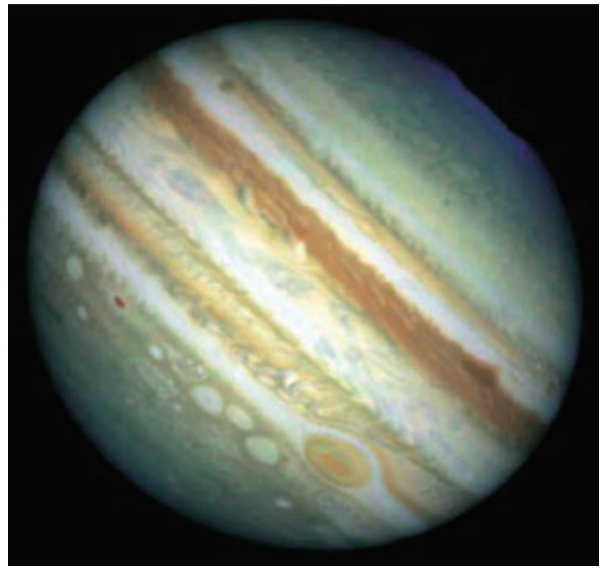

Fig. 3. The Jovian atmosphere observed by the spacecraft Voyager.

physics and mathematics is the formation of ordered or coherent structures that appears in temporal (or spatial) evolution of disturbances in nonlinear continuous media. Examples presented in this paper are formation of optical solitons in dielectric fibers and of coaxial zonal flows in a cylindrical plasma in magnetic field. Compatibility of these phenomena with the entropy law of thermodynamics is discussed. In case of soliton formation, the infinite numbers of conservations intrinsic to the evolution equation assures the integrability and exact causal relation between the initial condition and the final states. Thus the entropy has no room to increase. In case of turbulence self-organizations, additional conserved quantities, such as squared vorticity (called the enstrophy) is shown to be responsible to the increase of the system entropy and as a result, the energy can achieve an ordered structure.

Although exact (in case of solitons) and plausible (in case of turbulence self-organizations) mathematical descriptions have been derived for these cases, high speed computers developed in the later half of the 20th century have contributed fundamentally to these discoveries.

\section{References}

1) Hasegawa, A. and Tappert, F. D. (1973) Transmission of stationary nonlinear optical pulses in dispersive dielectric fibers. I. Anomalous dispersion. Appl. Phys. Lett. 23, 142-144.

2) Zakharov, V. E. and Shabat, A. B. (1972) Exact theory of two dimensional self-focusing and one dimensional self-modulation of waves in nonlinear media. Sov. Phys. JETP 34, 62-69.

3) Zabusky, N. J. and Kruskal, M. D. (1965) Interaction of solitons in a collisionless plasma and the recurrence of initial states. Phys. Rev. Lett. 15, 240-243.

4) Korteweg, D. J. and DeVries, G. (1895) On the change of form of long waves advancing in a rectangular canal, and on a new type of long stationary waves. Philos. Mag. Ser. 5 39, 422443.

5) Gardner, C. S., Greene, J. M., Kruskal, M. D. and Miura, R. M. (1967) Method of solving the Korteweg-deVries equation. Phys. Rev. Lett. 19, 1095-1097.

6) Lax, P. D. (1968) Integrals of nonlinear equation of evolution and solitary waves. Comm. Appl. Math. 21, 467-490.

7) Zakharov, V. E. and Shabat, A. B. (1972) Exact theory of two-dimensional self-focusing and onedimensional self-modulation of waves in nonlinear media. Sov. Phys. JETP 34, 62-69.

8) Kolmogorov, A. N. (1941) Local structure of turbulence in an incompressible viscous fluid at very high Reynolds numbers. Dokl. Akad. Nauk SSR 30, 299-303.

9) Kraichnan, R. H. (1967) Inertial ranges in two dimensional turbulence. Phys. Fluids 10, 14171423.

10) Hasegawa, A. (1978) Self-organization processes in continuous media. Advances in Phys. 34, 1-42.

11) Lilly, D. K. (1969) Numerical simulation of two dimensional turbulence. Phys. Fluids 12, II-240249.

12) Hasegawa, A. and Mima, K. (1978) Pseudo-threedimensional turbulence in magnetized nonuniform plasma. Phys. Fluids 21, 87-92.

13) Hasegawa, A. and Wakatani, M. (1987) Self-organization of electrostatic turbulence in a cylindrical plasma. Phys. Rev. Lett. 57, 1581-1584.

14) Hasegawa, A. (1983) Test of self-organization hypothesis in Jovian and Saturnian Wind Systems. J. Phys. Soc. Japan 52, 1930-1934.

(Received Sept. 30, 2008; accepted Dec. 1, 2008) 


\section{Profile}

Akira Hasegawa was born in 1934, graduated from the Osaka University in 1957 and obtained his $\mathrm{Ph}$. D degree from the University of California, Berkeley in 1964. He is an expert in nonlinear optics, plasma physics and comparative Japanese culture. He discovered the optical soliton and derived the master equation for information transfer in optical fibers. He also derived the kinetic Alfvén wave, the Hasegawa-Mima and Hasegawa-Wakatani equations and discovered the zonal flow in magnetized plasmas, as well as the dipole plasma container. Dr. Hasegawa is a recipient of the 1991 Rank Prize (UK), the 1995 Da Vinci Excellence Prize (France), the 1995 C\&C Prize (Japan), the 1998 IEEE/LEOS Quantum Electronics Award (US), the 2000 James Clerk Maxwell Prize of the American Physical Society and the 2008 Japan

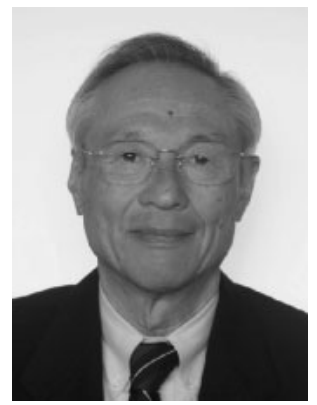
Academy Prize. He served as Distinguished Member of Technical Staff of Bell Laboratories, Professor of the Osaka University, Adjunct Professor of Columbia University, Visiting Professor at École Polytechnique Fédérale de Lausanne, Heinrich-Heine-Universität Düsseldorf, and at the Kobe Women's University. He is Fellow of the American Physics Society and of the Institute of Electrical and Electronics Engineering. Currently Dr. Hasegawa is Professor Emeritus of the Osaka University, Visiting Professor at Tianjin University and Zhejinag University in China and a member of the Kyoto East Rotary Club. 